\title{
Social and Political Context of Summer Camps in Occupied Palestine: A Brief Commentary
}

\author{
Amira Oudeh $^{1 *}$, lan Barron ${ }^{2}$, Ghassan Abdallah $^{3}$, Tahmina Nizam ${ }^{4}$ and Peter Willatts ${ }^{2}$ \\ ${ }^{1} \mathrm{NHS}$ Lothian, United Kingdom \\ 2University of Dundee, United Kingdom \\ ${ }^{3}$ Centre for Applied Research in Education, Ramallah, Palestinian Territory, United Kingdom \\ ${ }^{4}$ Assessment and Reconnection Division, London, United Kingdom
}

\begin{abstract}
The unique social and political context of the occupied Palestinian Territories (oPT) has several implications for conducting research there. The cumulative violence of decades of military occupation has resulted in intergenerational psychological disorders. Due to the lack of funding and poor economic climate, Palestinians seek psychological treatment through communal and creative outlets. One common method Palestinian communities use is summer camps, held across the oPT for children. We investigated the cognitive and emotional responses of children attending summer camps, to explore how effective these camps are in alleviating symptoms of trauma and stress. The current commentary highlights the importance of taking into account the social and political context in which summer camps are delivered. Factors identified for analysis include the geographical situation and the relationship to military violence, the nature and impact of 'area' administrative control, the extent of poverty and dependence on aid, and the local decision-making on the purpose and activities of camps as well as the selection criteria for children who attend camps. Recommendations are provided for future research.
\end{abstract}

\section{Keywords: Trauma; Emotion; Cognition; Summer camps}

The nationality "Palestinian" applies to people who resided in the British Mandate Palestine, before the creation of Israel in 1948, and to their descendants' post-1948. In 1967, Palestinians were given the territories of the Gaza Strip, the West Bank and East Jerusalem, following the Arab-Israeli Six Day War. These territories have been illegally occupied by Israel, and have been subject to political and military violence ever since. They are referred to as the occupied Palestinian Territories [1].

The continuous military violence has caused severe mental health problems for Palestinians living in the oPT [2]. Examples of conflict include injuries from firearms and explosives, abuse from illegal settlers, administrative detention, humiliation at checkpoints, and the separation wall (still in construction) in the form of an 8 meter tall concrete wall around the West Bank [3].

The separation wall has cut off agricultural land from farmers, disconnected Palestinian towns and villages, and stops the right of movement for Palestinians to travel between areas. The prohibition of right of movement is reinforced through Israeli military incursions and restrictions, inducing a severe limitation on fuel, water and electricity supplies, as well as sanitation issues. Consequently, Palestinians suffer from one of the world's most severe water shortages [4]. The wall is accompanied by 600 checkpoints in the West Bank which also inhibit Palestinians' right of movement to visit loved ones, work, educational institutions, Holy sites and health-care facilities [5].

The combination of the separation wall, checkpoints and military violence means that Palestinians in the oPT live in constant fear and anxiety for their own and others' wellbeing [6]. There is a general, negative psychological feeling reported around the oPT with Palestinians reportedly feeling hopelessness, anger, bereavement and helplessness [7]. The local Arabic term 'hamm', meaning to feel uneasy, anxiety and sadness [8] captures this experience [1].

Psychological problems are extremely common in individuals who have been subjected to at least one major traumatic event, such as a house incursion or witnessing someone's death [3]. Having repeated exposure to factors relating to a traumatic experience will reinforce feelings of fear or distress. For example, the Gaza Community Mental
Health Programme has stated that continued exposure to sonic booms from Israeli air force jets has presented symptoms similar to PTSD in Gazan Palestinians including feelings of fear, loss of concentration and appetite, bedwetting and stomach aches [4]. Being exposed to these factors repeatedly is detrimental to one's wellbeing; the living environment in the oPT helps to reinforce this exposure through house incursions and demolitions, military attacks, checkpoints and the separation wall.

Depression has been found to be the most prevalent in adults and Posttraumatic Stress Disorder (PTSD) in children [3]. This is not surprising; for most children in the oPT, the military occupation and subsequent cumulative violence has been present since their birth and throughout their perinatal period [9]. Notably, patient data from Médecins Sans Frontières (MSF) found that almost half of the patients were children aged 4-14 years of age, demonstrating the high prevalence of psychological disorders in the younger Palestinian population [10]. For example, one study found that children in the West Bank had, on average, experienced eleven different types of war events, such as being detained, physical assault by Israeli military and/or witnessing the death of a family member, [11]. Thus, both intergenerational trauma and developmental trauma have been identified among the Palestinian population in the oPT, with children in particular experiencing symptoms including complicated grief, school under achievement and depression [6], problem behaviours and suicidal intentions [12], and self-harm, substance misuse and dissociation [13]. Trauma in childhood is also related to difficulties in developmental domains such

*Corresponding author: Amira Oudeh, Honorary Assistant Psychologist, 21A The Heathery, Dunfermline, Fife, KY118TS, Scotland, Tel: +44 7535673642; E-mail: amiraoudeh@hotmail.com

Received February 01, 2016; Accepted February 18, 2016; Published February 25, 2016

Citation: Oudeh A, Barron I, Abdallah G, Nizam T, Willatts P (2016) Social and Political Context of Summer Camps in Occupied Palestine: A Brief Commentary. J Psychol Abnorm S1: 003. doi:10.4172/jpab.S1-003

Copyright: $\odot 2016$ Oudeh A, et al. This is an open-access article distributed under the terms of the Creative Commons Attribution License, which permits unrestricted use, distribution, and reproduction in any medium, provided the original author and source are credited. 
as delays in developmental skills, difficulties in cognitive functioning, and emotional and behavioural symptoms.

The alarming prevalence rates of psychological disorders in the oPT means that the majority of the Palestinian population need psychological intervention. However, the social and political climate of the oPT means that Palestinians lack the financial stability and infrastructure control required to develop professional psychological support programmes [3]. With the lack of mental health support through psychological therapies and medication, the rates of psychological disorders are alarmingly high. In particular, researchers have noted that services to support children are particularly scarce [6]. This is despite findings that highlight a marked improvement in mental health following psychotherapy and psychoeducation programs. The implications of a poor economic system mean that only small-scale therapeutic services are available to those living in the oPT [14]

As a solution to the lack of funding for therapy, community-oriented approaches have been developed to support individuals suffering from mental health disorders. This unique alternative fits well into the social and political context of Palestine, which has a culturally collectivist community across the oPT. Collectivism is a culture which emphasises an individual's identity as part of a group of community, typically found in Eastern societies. It particularly focuses on working together as a team to attain the groups' goals [15], thus it is not surprising that despite the high prevalence rates of mental health issues, Palestinians express a profound pride, aligning with the popular Palestinian ideology that 'to exist is to resist' [16-18]. In Palestine, this attitude is common, where the sense of self is linked in with family and community identity; that as long as the community continues to thrive under the occupation, they are resisting in every sense. The collectivist culture is known to be central as an emotional and psychological support base for Palestinians, particularly when a disaster happens such as a house demolition or the killing of a loved one [1]. As the difficult living circumstances are experienced by all, people are more understanding of events, can sympathise and are eager to help each other. This helps the sustainment of communal survival and high community cohesion of the Palestinian people as well as strengthening the resiliency of Palestinian families [7].

In Palestinian society, the extended family and neighbours play a key role in offering and providing support during times of distressing situations [19]. For example, when ambulances cannot get to patients due to roadblocks or checkpoints, members of the community will transport the patients [4]. This support provides families with coping mechanisms and becomes a protective factor that safeguards the traumatic experience from individuals [20].

It must be noted, however, that the occupation still affects the extended family too through emotional, economic and social damage [6]. An example of this is recurrent violent family home intrusions (a common occurrence in the oPT), where family members are at risk of being killed, physically or emotionally abused or arrested. There have been instances where parents are forced to watch their children be abused by the Israeli military. This in turn can trigger extreme feelings, thoughts and/or symptoms in parents that are linked to guilt, psychological disorders and more, thus potentially causing negative consequences on intra-family relations. For example, parents can become excessively overprotective, be emotionally and/or cognitively unavailable, and vent their anger on their children [7].

Such repercussions can be damaging to a child's recovery from traumatic experiences, as shown in research on the consequences of maternal PTSD and children after 9/11 [21]. It was found that if parents had a higher amount of mental health symptoms, then their children would be experiencing more psychological problems too. Collectively, research on families and trauma suggests that if a child is not protected during the time of the event by efficient caregivers, he or she may be more vulnerable to developing psychological problems from the event [22].

In saying this, such situations can sometimes sustain support, solidarity and cohesion between and within families. It has been found that youth, in particular, benefit from a strong network of support by peers and family, reinforced through schools and clubs [4]. This helps them keep a sense of hope for the future, which in turn keeps them from developing negative appraisals of the world, a tendency in patients of mental health problems like PTSD [23]. Additionally, studies have found that there were less psychological disorders presented than expected in children during times of uprisings, and it was proposed this may have been due to the adult community playing a protective factor in this [24].

By having a strong collectivist culture, and by offering additional support through community services, like summer camp clubs, Palestinian youth can be given the opportunity to recover from traumatic experiences they may have suffered under the occupation. Structured activities, provided through summer camps and other clubs, have been found to have a key role in supporting children's resiliency in traumatic situations [25]. Structured activities are thought to grant children with a scheduled routine, which can attempt to reinstate a sense of normality; give an opportunity to express and resolve problems; reduce the chance of exposure to dangerous environments; and to develop attachment and trust with other children and adults [26].

Research by Loughry and her team investigated the impact of structured activities on Palestinian children during times of difficulty. International NGOs (non-government organisations) during the second intifada developed child-focused interventions to support children's resiliency, through the use of structured activities. These were recreational and cultural activities such as sports, drama, art and traditional dancing. Loughry and colleagues' research aimed to increase resiliency by establishing normality as much as possible through community engagement. It was found that intervention was successful in rectifying children's behavioural and emotional well-being, and was connected to heightened caregiver support. A similar programme in a post-conflict zone of Uganda, offering activities like music, art and drama has also been found to positively impact children's psychosocial wellbeing [27].

As opposed to therapeutic approaches, where facilitators require specialised training, summer camps can be facilitated by community members such as teachers, community workers and volunteers. In the oPT, some summer camps are run by the National Committee for Sumer Camps (NCSC) and others by NGOs [14]. The results presented in the research literature propose that summer camps can be a successful method to alleviate at least some of the mental health difficulties Palestinian children have, and to encourage a positive and healthy development throughout the years [27]. Thus, our previous study sought to hear Palestinian children's evaluation of summer camps, in order to explore how effective these camps are, and how they could possibly be improved even more [14].

Our results presented differences between all participating in camps, due to each area having different political and social contexts. Indeed, Haj-Yahia states that differences in each community's difficulties therefore means that results cannot be generalised to the entire Palestinian population [7]. In our study, the participating camps were all from the Ramallah and East Jerusalem districts but all had 
very different experiences of the occupation. For example, Qatanna is encompassed by the separation wall, with only one entry and exit into the town. It was reported by one citizen that there is no outdoors area for children to play in because the wall is too close to the town. Qalandia is another town which is right beside the main checkpoint into the West Bank, and has been subjected to violent episodes such as Israeli military attacks [28]. Our study's results suggest that the political differences in each area may have an effect on children's emotional and cognitive development in a conflict environment. Qalandia is a zone A area, controlled by the Palestinian National Authority (PNA); and Qatanna is half zone B (controlled by the PNA and Israeli military) and half zone $\mathrm{C}$ (the Israeli military). The authority in control has an impact on the security and social development of the areas [1] and thus, this may explain some of the differences in results. Additionally, it may also be due to the different conflicts each town experiences; both Qatanna and Qalandia, as explained above, indicate contrasting traumatic exposure under the occupation. This suggests that the summer camp progamme may need to be more tailored to each area, and emphasises the individual differences between towns in the West Bank. Future research should consider investigating how area can affect children's emotional and cognitive well-being in further detail. This can be done by including a wider variety of towns from the West Bank, perhaps from different districts such as Tulkarem, Jenin, Nablus and Hebron.

As well as area differences, all camps were conducted in various ways. They all varied in size, activities, facilities and employee numbers. Palestinian culture has a large emphasis on gender roles, thus it is important to take note of the participating camps which had mixed sex attendance, and others had groups for each sex. Palestinian women are often seen to be the primary caregivers; generally they adopt the role of the housewife, whereas men are traditionally the bread winners of the family [4]. Our results found that sex had no effect on our study's results [14], which suggests that despite the clear gender roles set in Palestinian culture, there is no effect of it in children's emotional and cognitive functioning when considering summer camp attendance. Methodological limitations of the study would however leave such a conclusion tentative.

Both non-NCSC and NSC summer camps have similar overall aims: to develop children's cognitive, social and emotional capacity to live under occupation. This supports the idea that structured activities are a key to developing a child's resiliency [25-27]. Every participating summer camp offered arts and crafts, and sports, with Qalandia offering IT additionally. Both Kufr Ni'meh and Qatanna included creative writing and health classes, and Qatanna also had classes in culture, dancing, chess and fashion. Jericho had a particular focus on humanitarian and children's rights, history and first aid. When prompted on what their summer camps aims were in our qualitative measure, one camp worker in Qatanna remarked that the summer camp keeps the children occupied in doing something productive, and keeps them out of harm's way.

\section{Limitations}

The findings of the Oudeh et al study need to be viewed cautiously however [14]. The study was exploratory in nature with comparative groups rather than a randomised control design. The summer camps were volunteers and the standardised measure used was experimental in nature and translated for the first time into Arabic. Although there were qualitative questionnaires, a further standardised measure would have strengthened the triangulation of data. A narrow range of emotional and cognitive responses to traumatisation was assessed. Conclusions based on the empirical findings are therefore tentative.

\section{Conclusion}

In assessing the impact of summer camps on children's mental health in the occupied Palestinian territories, the current commentary highlights the importance of taking into account the historical, social and political context in which summer camps are delivered. Factors identified for analysis include the geographical situation and the relationship to military violence, the nature and impact of administrative control, the extent of poverty and dependence on aid, and the local decision-making on the purpose and activities of camps as well as the selection criteria for children who attend camps. In addition, future studies will need to assess the nature and extent of cumulative and ongoing traumatic events for children as well as for their families and communities. Such events include not only the military violence but also daily discrimination and humiliation at checkpoints, limitations on movement and the unpredictability of access to basic supplies such as water, health and electricity. Evaluation of summer camps will need to address the impact of camps on the resultant severe and wide ranging mental health symptoms, ensuring a broad range of symptoms are assess prior to and after camp attendance.

\section{Recommendations for Future Research}

Research into the effectiveness of summer camps needs to explore the interactive factors of geographical situation, military violence, administrative control, poverty, and dependency on aid as well as camp specific factors such the purpose, structure, activities and selection criteria of camps. More robust randomised control designs are needed with a range of standardised measures assessing a variety of mental health conditions. Qualitative studies could explore unique camp experiences for children in their own words. Longitudinal studies would be helpful in assessing the long term impact of repeated camp attendance over the years.

\section{References}

1. Giacaman R, Khatib R, Shabaneh L, Ramlawi A, Sabri B, et al. (2009) Health status and health services in the occupied Palestinian territory. Lancet 373 : 837-849.

2. Giacaman R, Husseini A Gordon NH, Awartani F (2004) Imprints on the consciousness: the impact on Palestinian civilians of the Israeli Army invasion of West Bank towns. Eur J Public Health 14: 286-290.

3. Espie E, Gaboulaud V, Baubet T, Casas G, Mouchenik Y, et al. (2009) Traumarelated psychological disorders among palestinian children and adults in Gaza and West Bank, 2005-2008. International Journal of Mental Health Systems 3: $1-5$

4. Batniji R, Rabaia Y, Nguyen-Gillham V, Giacaman R, Sarraj E, et al. (2009) Health as human security in the occupied Palestinian territory. Lancet 373 : 1133-1143.

5. Khalidi R, Samour S (2011) Neoliberalism as liberation: The Statehood programme and the re-thinking of the national Palestinian movement. Journal of Palestine Studies 40: 6-25.

6. Barron I, Abdallah G (2015) Intergenerational Trauma in the Occupied Palestinian Territories. Submitted to the Journal of Child and AdolescentTrauma 8: 103-110.

7. Haj-Yahia MM (2007) Challenges in studying the psychological effects of Palestinian children's exposure to political violence and their coping with this traumatic experience. Child Abuse Negl 31: 691-697.

8. Owais $Y(2010)$ The Supplication Series: Distress, Sadness and Anxiety.

9. Svraka E, Loga S (2006) [War psychotrauma of pregnant women as prenatal factor of cerebral palsy]. Med Arh 60: 376-378.

10. Gaboulaud V, Reynaud C, Moro MR, Roptin J, Lachal C, et al. (2010) Psychological support for Palestinian children and adults: An analysis of data from people referred to the Medecins Sans Frontieres Programme for behavioural and emotional disorders in the occupied Palestinian territory. Intervention 8: 131-142. 
Citation: Oudeh A, Barron I, Abdallah G, Nizam T, Willatts P (2016) Social and Political Context of Summer Camps in Occupied Palestine: A Brief Commentary. J Psychol Abnorm S1: 003. doi:10.4172/jpab.S1-003

11. Barron I, Abdullah G, Smith P (2013) Randomised Control Trial of a CBT Recovery Program in Palestinian Schools. Journal of Loss and Trauma: International Perspectives on Stress and Coping 18: 306-321

12. Kilmer RP, Gil-Rivas (2010) Exploring posttraumatic growth in children impacted by Hurricane Katrina: Correlates of the phenomenon and developmental considerations. Child Development 81: 1211-1227.

13. Punamäki R, Quota S, El Sarraj E (2001) Resiliency factors predicting psychological adjustment after Palestinian violence among Palestinian children. International Journal of Developmental Behavior 25: 256-267.

14. Oudeh A, Nizam T, Barron I, Abdallah G, Willatts P (2014) Emotional and Cognitive Responses of Children Attending Summer Camps in Occupied Palestine: A Pilot Study. Journal of Psycholological Abnormalities in Children 4: 136.

15. Triandis HC (1996) The psychological measurement of cultural syndromes American Psychologist 51: 407-415.

16. Kanzi R (2009) To Exist is to Resist.

17. Bullimore K (2011) 'Sumoud' in Palestine: To Exist is to Resist.

18. Tuhus M (2012) In Palestine, to Exist is to Resist.

19. Chatty D, Hundt G, Damaj M (2001) Children and adolescents in Palestinian households: Living with the effects of prolonged conflict and forced migration. Lessons Learned Report: 1-34.

20. Quota S, Punamaki R-L, Sarraj E (2008) Child development and family mental health in war and military violence: The Palestinian experience. International Journal of Behavioural Development 32: 310-321.

21. Chemtob CM, Nomura Y, Rajendran K, Yehuda R, Schwartz D, Abramovitz $R$ (2010). Impact of maternal posttraumatic stress disorder and depression following exposure to the September 11 attacks on preschool children's behaviour. Child Development 81: 1129-1141.

22. Masten AS, Osofsky JD (2010) Disasters and their impact on child development introduction to the special section. Child Dev 81: 1029-1039.

23. Bryant RA, Salmon K, Sinclair E, Davidson P (2007) A prospective study of appraisals in childhood posttraumatic stress disorder. Behav Res Ther 45 2502-2507.

24. Baker A, Shalhoub-Kevorkian N (1999) Effects of political and military traumas on children: the Palestinian case. Clin Psychol Rev 19: 935-950.

25. Aguilar P, Retamal G (1998) Rapid Educational Response in Complex Emergencies: A Discussion Document. Geneva: UNESCO, UNICEF \& UNHCR.

26. Loughry M, Ager A, Flouri E, Khamis V, Afana AH, et al. (2006) The impact of structured activities among Palestinian children in a time of conflict. J Child Psychol Psychiatry 47: 1211-1218.

27. Ager A, Akesson B, Stark L, Flouri E, Okot B, McCollister F, et al. (2011) The impact of the school-based psychosocial structured activities (PSSA) program on conflict-affected children in Northern Uganda. Journal of Child Psychology and Psychiatry 52: 1124-1133.

28. Mousa H (2011) 5 Injured in Demonstrations at Qalandia Checkpoint. 